\title{
An unusual organism causing orbital cellulitis
}

\author{
HAROLD SCHWARTZ, ${ }^{1}$ MARK A. BASKIN, ${ }^{1}$ ANDREW ILKIW, ${ }^{2}$ AND \\ LEON LEBEAU ${ }^{3}$ \\ From the ${ }^{1}$ Departments of Ophthalmology and ${ }^{2}$ Otolaryngology, University of Illinois Abraham Lincoln \\ School of Medicine, and the ${ }^{3}$ Bacteriology Section, University of Illinois Hospital Laboratories, Chicago
}

SUMMARY Bacterial orbital cellulitis is a feared complication of paranasal sinus infection. Staphylococcus and Streptococcus species are the commoner pathogens involved in these cases. However, anaerobic bacteria and unusual Gram-negative organisms should be suspected as well. We treated a case of bacterial orbital cellulitis due to foci of infected paranasal sinuses caused by Eikenella corrodens, a Gram-negative rod. The patient was managed with intensive antibiotic coverage and surgical intervention.

Bacterial orbital cellulitis may endanger sight as well as life. Its primary cause is an extension of pathogens from infected paranasal sinuses (Smith and Spencer, 1948; Haynes and Carmblett, 1967; Jarret and Gutman, 1969; Chandler et al., 1970). Thus, the organisms commonly responsible for sinus infection, Staphylococcus aureus and Streptococcus species, are the most common aetiological agents involved in orbital cellulitis (Haynes et al., 1967; Jarret et al., 1969). Treatment is based on intensive antibiotic therapy with adjunctive surgical intervention. This report describes a case of orbital cellulitis caused by an unusual bacterial organism that was successfully treated in that way.

\section{Case report}

A 19-year-old white man was referred to the University of Illinois Eye and Ear Infirmary after a 2-day history of progressive pain, swelling, and redness of his left upper and lower eye lids. The patient had sought medical attention the day before admission, and had received $300 \mathrm{mg}$ clindamycin phosphate intramuscularly. The patient reported a 5 -year history of intermittent sinus infections that had been treated with oral ampicillin sulphate. Two weeks before the onset of the present symptoms the patient had used ampicillin sulphate for a coldlike illness characterised by running nose and low fever. He denied trauma or previous ocular surgery.

Physical examination at the time of admission revealed severe swelling of the left upper and lower

Address for reprints: Dr H. Schwartz, Illinois Eye and Ear Infirmary, 1855 West Taylor Street, Chicago, Illinois, USA eyelids with marked induration and erythema. A sharp demarcation line was noted in the left upper eyelid between erythematous and non-erythematous tissue. Marked proptosis was present. The best corrected visual acuity was 20/20 OD and 20/40 OS. The right eye was unaffected. The conjunctiva of the left eye displayed $3+$ injection, with chemosis. The cornea, anterior chamber, and pupillary response were normal. Severe limitation of motility was observed. The fundus was normal.

Crepitation of the left ethmoidal area was found. The remainder of the physical examination was within normal limits.

Sinus $x$-ray films obtained on admission (Fig. 1) showed clouding of the ethmoid and maxillary sinuses. A Gram stain of nasal secretion revealed no organisms. The leukocyte count was $10500 / \mathrm{mm}^{3}(10 \cdot 5$ $\left.\times 10^{9} / 1\right)$ with a marked left shift. The patient was started on a regimen of intravenous methicillin sodium, $2 \mathrm{~g}$ every 4 hours, which was alternated with 3 million units of intravenous penicillin every 4 hours. Twelve hours after admission his condition had not improved, and an external and internal ethmoidectomy and maxillary antrostomy were performed.

A curved incision $4 \mathrm{~cm}$ long was made above the medial canthus, laterally along the nasal bridge, and extended to the supraorbital rim below the level of the eyebrow. The incision was carried through the periosteum to the bone. The periosteum was raised to expose the area of the orbital plate of the frontal bone. With a feeler elevator the eroded medial orbital wall was entered, and approximately $10 \mathrm{ml}$ of pus was aspirated and taken for Gram stain and anaerobic cultures. An intranasal ethmoidal ap- 


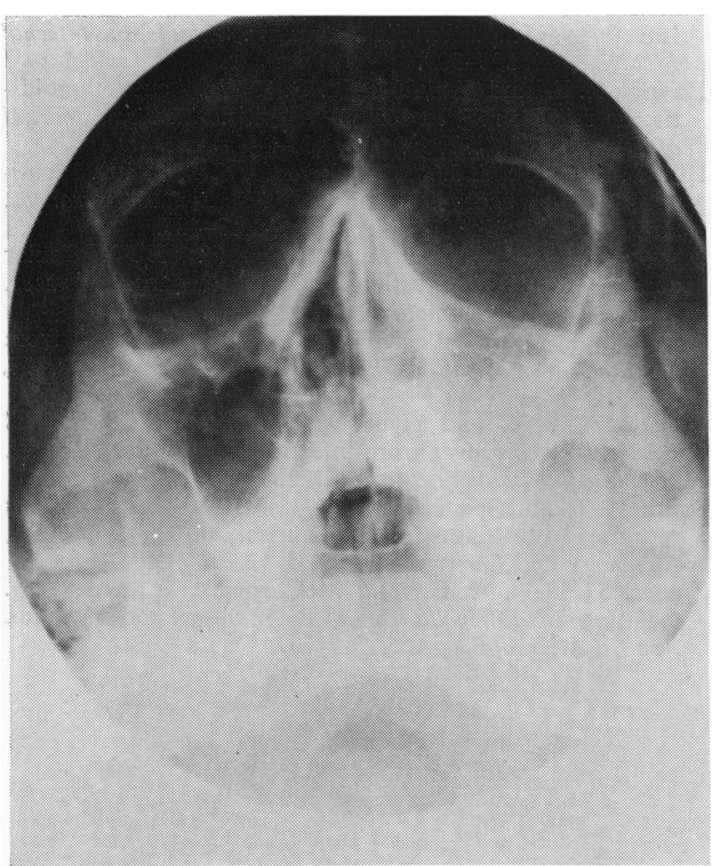

Fig. 1 Sinus x-ray film taken at admission reveals diffuse soft tissue swelling in region of left cheek and clouding of ethmoid and maxillary sinuses

proach was then performed lateral to the middle turbinate.

When the medial ethmoid cells were opened, additional pus was aspirated. Finally, by placing a small trocar beneath the left inferior turbinate, an intranasal antrostomy was done and the maxillary antrum was entered. A small rubber drain was sutured into place to drain the maxillary antrum. The external incision was loosely closed in layers with a $1-\mathrm{cm}$ rubber drain placed in position.

A Gram stain of the aspirated material disclosed predominantly Gram-positive cocci suggestive of Streptococcus, although Staphylococcus could not be ruled out. No Gram-negative organisms were seen. The patient was continued on the same preoperative antibiotic regimen. On the third postoperative day the organism was characterised.

The organism, which was isolated on blood agar and isovitalex-enriched chocolate agar, grew so slowly that in $\mathbf{7 2}$ hours the dry colonies were only $0.5 \mathrm{~mm}$ or less in diameter. Within 5 to 7 days typical growth of pitting colonies appeared. Gram stains from culture plates done at 72 hours revealed a thin Gram-negative rod, $0.5 \times 1.5 \mu \mathrm{m}$. Biochemical analyses showed the organism to be positive for lysine, ornithine, oxidase, and nitrate, and negative for arginine, catalase, and indole. It did not ferment carbohydrates. In disc antibiotic susceptibility studies the organism was seen to be susceptible to ampicillin, cephalothin, tetracycline, penicillin, and erythromycin, and resistant to oxacillin and clindamycin. From these studies the organism was classified as Eikenella corrodens (Hill et al., 1970). Staphylococcus epidermidis was also isolated.

On the basis of the antibiotic sensitivity studies the patient was given 4 million units of intravenous penicillin every 6 hours, and the methicillin sodium was discontinued. By the fourth postoperative day he had regained full motility. No evidence of proptosis or conjunctival chemosis was noted. Minimal eyelid oedema persisted, but was not visible after 2 weeks of antibiotic therapy, after which the patient was discharged.

\section{Discussion}

Eikenella corrodens, a facultive Gram-negative rod, has only recently been implicated as a human pathogen. To our knowledge this organism has never been isolated from ocular tissue, though it has been reported from numerous and diverse clinical sources (Zinner et al., 1973).

This organism has been isolated in 16 of 64 positive blood cultures from 100 patients taken 1 minute after tooth extraction (Khairat, 1967). In a series of 6 children it was detected in subdural empyema and brain abscess secondary to frontal sinus infection (Marsden and Hyde, 1971). Additional infections noted in the latter series included abdominal abscess, otitis media, and a reaction from a human bite. The organism was isolated in pure culture in 17 cases of infected frontal sinuses, wounds, and peritoneal fluid, and in mixed flora from abscesses and sputum (Bottone et al., 1973).

The most recent report describes 7 of 10 cases in which Eikenella corrodens was isolated in pure culture from abdominal abscess, lung empyema, meningitis, and endocarditis (Dorff et al., 1974). It appears to reside in the oropharynx and the gastrointestinal tract. The paranasal sinuses may become involved if chronic infection is left untreated.

Our patient developed orbital cellulitis from a focus of chronic paranasal sinus infection. Although Gram stain of material obtained intraoperatively suggested Staphylococcus or Streptococcus, cultures grew only Staphylococcus epidemidis as a contaminant. No suspicion of Gram-negative infection was present until the culture results were available. Since this case was seen an additional case of orbital cellulitis caused by Eikenella corrodens has been observed at our institution. This case was due to an infected orbital prosthesis in a patient who had undergone orbital exteneration. 
When investigating organisms causing orbital cellulitis as a result of chronically infected sinuses anaerobic bacteria and unusual Gram-negative pathogens as well as the more common bacterial agents must be considered. We recommend the use of a broad spectrum of antibiotics such as gentamycin and penicillin or methicillin and penicillin until definite culture results are received, for some organisms may not be identified until after a long (3-day) incubation.

\section{References}

Bottone, E. J., Kittick, J., Jr., and Scheirson, S. S. (1973). Isolation of Bacillus $H B-1$ from human clinical sources. American Journal of Clinical Pathology, 59, 560-564.

Chandler, J. R., Langenbrunner, D. J., and Stevens, E. R. (1970). The pathogenesis of orbital complication in acute sinusitis. Laryngoscope, 80, 1-12.

Dorff, G. J., Jackson, L. J., and Rytel, M. W. (1974).
Infections with Eikenella corrodens. Annals of Internal Medicine, 80, 305-309.

Haynes, R. E., and Carmblett, H. G. (1967). Acute ethmoiditis: its relationship to orbital cellulitis. American Journal of Diseases of Children, 114, 261-265.

Hill, L. R., Snell, J. J. S., and Lapage, S. P. (1970). Identification and characterization of Bacteroides corrodens. Journal of Medical Microbiology, 3, 483-487.

Jarrett, W. H. III, and Gutman, F. A. (1969). Ocular complications of infections of the paranasal sinuses. Archives of Ophthalmology, 81, 683-689.

Khairat, O. (1967). Bacteroides corrodens isolated from bacteraemias. Jornal of Pathology and Bacteriology, 94, 29-35.

Marsden, H. B., and Hyde, W. A. (1971). Isolation of Bacteroides corrodens from infections in children. Journal of Clinical Pathology, 24, 117-122.

Smith, A. T., and Spencer, J. T. (1948). Orbital complications resulting from lesions of the sinuses. Annals of Otology, Rhinology and Laryngology, 57, 5-12.

Zinner, S., Daly, A. K., and McCormack, W. M. (1973). Isolation of Eikenella corrodens in a general hospital. Applied Microbiology, 25, 705-709. 